La gestión de los ingresos y su incidencia en la inversión pública del Gobierno Autónomo Descentralizado Municipal de Salinas (GADMS), período 2010 - 2016.

Margarita Panchana P., Hermelinda Cochea T., Víctor Gómez P.

Recibido: noviembre 2016

Aprobado: mayo 2017 


\title{
La gestión de los ingresos y su incidencia en la inversión pública del Gobierno Autónomo Descentralizado Municipal de Salinas (GADMS), período 2010 - 2016
}

\section{The revenue management and its impact on the public investment of the Decentralized Municipal Government of Salinas (GADMS), period 2010 -}

\author{
2016 \\ Margarita Panchana Panchana, Hermelinda Cochea Tomalá, Víctor Gómez Panchana \\ Facultad de Ciencias Administrativas \\ Universidad Estatal Península de Santa Elena \\ margaritapanchana@hotmail.com
}

\begin{abstract}
Resumen
La presente investigación se centra en el estudio y análisis de la gestión de los ingresos y su incidencia en la inversión del Gobierno Autónomo Descentralizado Municipal de Salinas GADMS, período 2010 - 2016, y se fundamenta en la Constitución de la República del Ecuador (2008), Plan Nacional del Buen Vivir, COOTAD, y Plan de Desarrollo y Ordenamiento Territorial del cantón Salinas. El tipo de investigación utilizado fue el descriptivo, los métodos que aportaron al estudio fueron analítico, sintético y reflexivo, y la técnica de análisis documenta; se revisó documentos y estudios relacionados con la gestión de los ingresos y la realidad de las necesidades básicas de la población del cantón Salinas. Los resultados indican que el GADMS carece de políticas idóneas de gestión de cobro y disciplina fiscal, situación que limitó la atención de las demandadas de sus parroquias. Se evidencia que la hipótesis planteada no permite satisfacer las necesidades básicas de la población; por lo tanto, es verdadera. En el 2016 se actualizó el catastro, sin embargo, los recursos fueron insuficientes para atender las necesidades registradas en el PDOT 2014 - 2019. La mayor recaudación de los ingresos de toda entidad pública de esta naturaleza, está en función de las iniciativas y grado de responsabilidad de los gerentes públicos y directivos, de las políticas internas de gestión de cobro que se implementen y de la dirección efectiva e integrada de los procesos de creación de valor público.
\end{abstract}

Palabras clave: GAD Salinas, gestión, presupuesto, inversiones, transferencias

\section{Abstract}

The present research focuses on the study and analysis of the income management and its impact on the investment of the Municipal Government Decentralized Salinas GADMS, period 2010 - 2016, and is based on the Constitution of the Republic of Ecuador (2008), National Plan of Good Living, COOTAD, and Plan of Development and Territorial Planning of the canton Salinas. The type of research used was descriptive, the methods that contributed to the study were analytical, synthetic and reflexive, and the document analysis technique, reviewed documents and studies related to income management and the reality of basic needs of the Population of the canton Salinas. The results indicate that GADMS lacks adequate collection and fiscal discipline management policies, which limited the attention of the defendants of their parishes. It is evident that the hypothesis proposed does not meet the basic needs of the population; Therefore, it is true. In 2016 the cadaster was updated; however, the resources were insufficient to meet the needs recorded in PDOT 2014 - 2019. The highest revenue collection of any public entity of this nature, is a function of the initiatives and degree of the responsibility of public managers and managers, the internal collection management policies implemented and the effective and integrated management of public value creation processes.

Keywords: GAD Salinas, management, budget, investments, transfers 


\section{Introducción}

La limitada gestión de las actividades generadoras de ingresos del Gobierno Autónomo Descentralizado Municipal de Salinas (GADMS), trae como consecuencia la insuficiencia del bien dinero frente a las demandas de los servicios básicos por parte de la población.

El objetivo de esta investigación fue analizar la incidencia de la gestión de las actividades generadoras de ingresos en las demandas de los servicios básicos de la población, mediante la aplicación de la técnica de análisis documental para la emisión de una opinión respecto a los resultados obtenidos.

El nuevo marco legal e institucional, en el cual se cobijan los gobiernos autónomos descentralizados, es el Código Orgánico de Ordenamiento Territorial, Autonomía y Descentralización, en el cual se transfieren competencias a los municipios y se establece una nueva forma de financiamiento para el funcionamiento de estos entes seccionales. Esta investigación, se justifica plenamente por la importancia de los ingresos del Gobierno Autónomo Descentralizado Municipal de Salinas (GAD), pretendiendo determinar si estos alcanzan para realizar las inversiones y proporcionar a la población los servicios básicos fundamentales, como: alcantarillado, recolección de basura, alumbrado eléctrico, entre otros, que al mismo tiempo sirven para el desarrollo de otras actividades productivas.

Según (Asamblea Nacional, 2008) Art. 238 de la Constitución de la República del Ecuador, los Gobiernos Autónomos Descentralizados gozarán de autonomía política, administrativa y financiera, y se regirán por los principios de solidaridad, subsidiariedad, equidad interterritorial, integración y participación ciudadana. En ningún caso el ejercicio de la autonomía permitirá la secesión del territorio nacional.

La (Asamblea Nacional, Constitución de la Repùblica, 2008) Artículo 238 segundo párrafo dice: constituyen gobiernos autónomos descentralizados las juntas parroquiales rurales, los consejos municipales, los consejos metropolitanos, los consejos provinciales y los consejos regionales. (pág. 46)

La (Corporación de Estudios y Publicaciones, Código Orgánico de Organización, Autonomía y Descentralización, 2012) Mediante el COOTAD, artículo 52, referente a la naturaleza jurídica de los Gobiernos Autónomos Descentralizados Municipales, dice que son personas jurídicas de derecho público, con autonomía política, administrativa y financiera, que estarán integrados por las funciones de participación ciudadana, legislación y fiscalización, y, ejecutiva previstas en este Código, para el ejercicio de las funciones y competencias que le corresponden.

(Herrera Library, 2007) sostiene que la Gestión para Resultados tiene la función de facilitar a las organizaciones públicas la dirección efectiva e integrada de su proceso de creación de valor público, a fin de optimizarlo asegurando la máxima eficacia, eficiencia y efectividad de su desempeño, la consecución de los objetivos de gobierno y la mejora continua de sus instituciones. (pág. 18)

(García Moreno \& García López, 2011) dicen que la Gestión para Resultados del Desarrollo en Gobiernos Sub-nacionales, es una estrategia que orienta las acciones de los actores públicos del desarrollo hacia la generación del mayor valor público posible (cambios sociales observables y susceptibles de medición), a través del uso de instrumentos de gestión que, en forma colectiva, coordinada y complementaria, deben implementar las instituciones públicas sub-nacionales y contribuir a la mejora de la calidad de vida de la población. (pág. 14).

(Castillo Peñaherrera, 2013) sostiene que la transparencia, innovación, conectividad tecnológica y control de calidad deberían ser cualidades inherentes de toda gestión. Además, afirma que la gestión de calidad, exige la aplicación de procedimientos con estándares internacionales de calidad en las instituciones públicas, sobre todo en sectores de Salud, Educación y Servicios, es algo de lo que no se puede prescindir. Añade, la idea es adaptarse a sistemas internacionales que se ajusten a la realidad de Ecuador. (pág. 1)

\section{Presupuesto}

La (Corporación de Estudios y Publicaciones, Código Orgánico de Planificación y Finanzas Públicas, Tomo III, 2012) Manual General de Contabilidad Gubernamental, dice que el Presupuesto es el instrumento de política fiscal en el cual constan las estimaciones de ingresos a obtener, así como los gastos que podrán realizarse en función del financiamiento previsto; es decir por una parte constan el origen de sus fuentes de financiamiento a través de la identificación de las diversas partidas tributarias y no tributarias y por otra el destino que se dará a los recursos financieros durante su vigencia.

(Guzman, 2011) dice que, en gestión pública, el concepto presupuesto por resultados o presupuesto basado en el desempeño se refiere al funcionamiento y los alcances de las acciones públicas. (pág. 22) 
(Paredes, 2006) afirma que el presupuesto público es un instrumento de gobierno que constituye el motor de la administración del Estado y, alrededor de él, se desenvuelve gran parte de la vida económica del país. Son limitadas las decisiones cotidianas de la política y administración que no estén vinculadas con los ingresos y egresos del presupuesto público. Entre las decisiones, se pueden mencionar las siguientes: 1) cuando el estado crea o suprime impuestos; 2) cuando crea o suprime cargos públicos; 3) cuando decide llevar un proyecto de inversión; 4) cuando aumenta las remuneraciones de los servidores públicos. Todas estas disposiciones influyen de distintas formas en el desarrollo de las actividades productivas y financieras del país. (pág. 36)

\section{Inversiones}

La (Corporación de Estudios y Publicaciones, Código Orgánico de Planificación y Finanzas Públicas, Tomo I, 2013) con respecto a las inversiones públicas, formula que es la principal expresión de las finanzas públicas, definidas como el "conjunto de egresos y/o transacciones que se realizan con recursos públicos" cuya ejecución se prevé en los "planes de inversión". La priorización de los programas y proyectos de inversión corresponde a la SENPLADES, a través de la inserción en el plan anual de inversiones del presupuesto general del Estado, de acuerdo a los lineamientos fijados en el COPLAYFP.

(Ecuador, 2011) describe que el país se encuentra en un proceso de cambio que requiere nuevos desafíos, la Constitución de la República, el Plan Nacional del Buen Vivir y las Políticas Públicas han restablecido la imp

ortancia de la inversión pública como variable instrumental para lograr los cambios estructurales que conduzcan a una sociedad más justa, solidaria y, sobre todo, en la que se pueda convivir con los demás y con la naturaleza, ejerciendo plenamente el buen vivir. Quedaron atrás los días en que la inversión pública se limitaba a las posibilidades de ajuste fiscal y la estabilización macroeconómica; y así la salud, la educación, la cultura, el ambiente entre otros ámbitos, quedaban relegados al cumplimiento de los llamados "equilibrios macroeconómicos". Por ello, ahora hay mayor responsabilidad para quienes proponen, desarrollan, gestionan, y ejecutan proyectos, programas de inversión pública. Se debe velar por la calidad de la inversión, uso oportuno y óptimo de los recursos fiscales, por la coherencia programática de las iniciativas, en el mediano y largo plazo; y sobre todo, cada ciudadano asegurarse de que estas inversiones sean altamente rentables desde el punto de vista económico, social o ambiental. (pág. 13)
$\mathrm{Su}$ devengamiento produce contablemente modificaciones directas en la composición patrimonial del Gobierno Autónomo Descentralizado Municipal de Salinas, aumentando sus inversiones en infraestructura física institucional o en bienes nacionales de uso público y en productos intangibles de desarrollo social; implica, además, la concesión de transferencias sin contraprestación destinadas a la inversión.

\section{El Buen Vivir}

Según la (Secretaría Nacional de Planificación y Desarrollo SENPLADES, 2013) El Buen Vivir, se planifica, no se improvisa, es la forma de vida que permite la felicidad y la permanencia de la diversidad cultural y ambiental, es la armonía, igualdad, equidad y solidaridad, no es buscar la opulencia ni crecimiento económico infinito. La planificación del Buen Vivir, como su línea rectora es contraria a la improvisación que genera enormes costos a una sociedad con escases de recursos.

\section{Materiales y métodos}

El tipo de investigación utilizada fue la descriptiva, los métodos que aportaron al estudio fueron el analítico, sintético y reflexivo, y con la ayuda de la técnica de análisis documental, se revisó documentos y estudios relacionados con la gestión de los ingresos y la realidad de las necesidades básicas de la población del cantón Salinas. Se realizó un conversatorio con las autoridades del GAD de Salinas, directos involucrados en la problemática del cantón.

Se revisó información presupuestaria y financiera del GAD de Salinas, el método histórico, inductivo y deductivo fue útil para aprobar la hipótesis planteada, rechazarla y/o aceptarla al término de la investigación.

\section{Hipótesis}

Las limitadas gestiones de las actividades generadoras de ingresos incidieron en la inversión del Gobierno Autónomo Descentralizado Municipal de Salinas.

Tabla 1 Ingresos efectivos 2010 - 2016

\begin{tabular}{|c|c|c|c|c|}
\hline \multirow{2}{*}{ Denominación } & \multicolumn{3}{|c|}{ Años } & \multirow{2}{*}{$\%$} \\
\cline { 2 - 4 } & $\mathbf{2 0 1 0}-\mathbf{2 0 1 5}$ & $\mathbf{2 0 1 6}$ & TOTAL & \\
\hline INGRESOS EFECTIVOS & 101.255 .982 & 18.425 .479 & 119.681 .461 & \multirow{2}{*}{100} \\
\hline
\end{tabular}

Fuente: cédulas presupuestarias del GAD Municipal Salinas

Los ingresos del GADMS provienen de impuestos prediales, tasas y contribuciones de mejoras, y otros, para el año 2016 generaron $\$ 18^{\prime} 425,478$, resultando 
insuficiente para cubrir la demanda de necesidades. El catastro estuvo sin actualizarse desde el 2007 hasta 2015, incumpliendo lo dispuesto en el artículo No. 139 del COOTAD, el cual señala que es obligación de los GAD actualizar cada dos años los catastros y valorar la propiedad urbana y rural. La tasa anual de funcionamiento de establecimientos turísticos (hoteles, hostales, pensiones, bares, discotecas, fuentes de soda, peñas, centros de convenciones, restaurantes, cafeterías, agencias de viajes y turismo), no ha sido actualizada desde el año 2003.

Tabla 2 Gastos corrientes en personal e inversión en obras públicas, en dólares, período 2010 - 2016

\begin{tabular}{|c|l|r|r|r|}
\hline \multirow{2}{*}{$\begin{array}{c}\text { Part. } \\
\text { Presup }\end{array}$} & \multicolumn{1}{|c|}{ Denominación } & \multicolumn{2}{|c|}{ Años } & \multirow{2}{*}{ Total } \\
\cline { 3 - 4 } & & $\mathbf{2 0 1 0}-\mathbf{2 0 1 5}$ & \multicolumn{1}{c|}{$\mathbf{2 . 0 1 6}$} & \\
\hline $\mathbf{5 1}$ & Gastos de personal & 15.176 .318 & 1.937 .224 & $\mathbf{1 7 . 1 1 3 . 5 4 2}$ \\
$\mathbf{7 1}$ & Gastos de personal e inversión & 29.125 .171 & 4.510 .980 & $\mathbf{3 3 . 6 3 6 . 1 5 0}$ \\
$\mathbf{7 5}$ & Obras públicas & 15.260 .644 & 3.409 .157 & $\mathbf{1 8 . 6 6 9 . 8 0 1}$ \\
\cline { 2 - 5 } & Suman Totales: .................. & $\mathbf{5 9 . 5 6 2 . 1 3 2}$ & $\mathbf{9 . 8 5 7 . 3 6 1}$ & $\mathbf{6 9 . 4 1 9 . 4 9 3}$ \\
\hline
\end{tabular}

Fuente: cédulas presupuestarias del GAD Municpal Salinas

El gasto corriente en personal durante el periodo 2010 - 2015 ascendió a \$15'176,318, representando el $14,99 \%$ de $\$ 101$ '255,982 total de ingresos para el mismo rango de años, y la inversión en obra pública equivale a $15,07 \%$ del ingreso efectivo, porcentaje diferente en 0,000833 puntos más - menos.

En el año 2016 se invirtió en gasto corriente de personal "GCP" el $11 \%$ del ingreso efectivo "IE" \$18’425,479 y para Obras Públicas “OP” se destinó el $19 \%$ del ingreso efectivo.

Tabla 3 Estructura de gasto de personal e inversión en dólares, período 2010 - 2016

\begin{tabular}{|c|c|c|c|c|}
\hline \multirow{2}{*}{$\begin{array}{l}\text { Part. } \\
\text { Presup }\end{array}$} & \multirow{2}{*}{ Denominación } & \multicolumn{2}{|c|}{ Años } & \multirow{2}{*}{ Total } \\
\hline & & 2010-2015 & 2.016 & \\
\hline & Ingreso efectivo & 101.255.982 & 18.425 .479 & 119.681 .461 \\
\hline 51 & Gastos corrientes en personal & 15.176 .318 & 1.937 .224 & 17.113.542 \\
\hline \multirow[t]{2}{*}{71} & Gastos de personal e inversión & 29.125 .171 & 4.510 .980 & 33.636 .150 \\
\hline & Sub - total $($ GCP + GPI) : ...... & 44.301.489 & 6.448 .204 & 50.749 .692 \\
\hline \multirow[t]{6}{*}{75} & Obras públicas & 15.260 .644 & 3.409 .157 & 18.669 .801 \\
\hline & Suman Totales: ........................ & 59.562 .132 & 9.857 .361 & 69.419 .493 \\
\hline & Coef.relac. GCP/IE & $15 \%$ & $11 \%$ & $14 \%$ \\
\hline & Coef.relac. GPI/IE & $29 \%$ & $24 \%$ & $28 \%$ \\
\hline & Coef.relac. GTP/IE & $44 \%$ & $35 \%$ & $42 \%$ \\
\hline & Coef.relac. OP/IE & $15 \%$ & $19 \%$ & $16 \%$ \\
\hline
\end{tabular}

Fuente: cédulas presupuestarias del GAD Municipal Salinas

En el periodo 2010-2015, el gasto de personal e inversión, ascendió al 29\% del ingreso efectivo para el mismo número de años, el cual es de $\$ 101$ '255,982. El gasto corriente en personal (Grup. 51) sumado al gasto personal e inversión (Grup. 71), es decir (15\%+ $29 \%$ ), representa el $44 \%$ del ingreso efectivo. En el año 2016 el gasto de personal e inversión $(\$ 4 ’ 510,980)$ sumado al gasto corriente en personal $(\$ 1,937,224)$, representa el $35 \%$ del ingreso efectivo del mismo año.

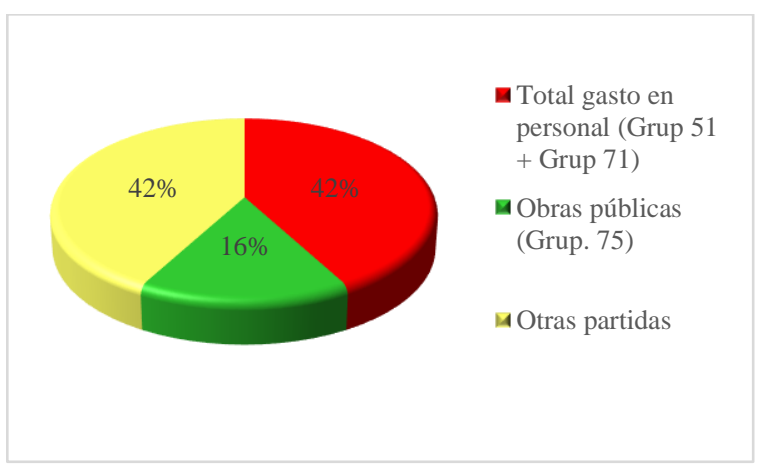

Figura 1 Distribución del gasto financiado con el ingreso efectivo

Del análisis comparativo entre los valores invertidos en obras públicas, periodo 2010 - $2016(16 \%)$ y el porcentaje total de gastos en personal $42 \%$, se determina que este último es mayor a la IOP.

Sumado el total de gasto en personal y la inversión en obra pública, durante el periodo 2010 - 2016 (42\% + $16 \%$ ) se tiene el $58 \%$ del ingreso efectivo (\$119’681,461) para el igual número de años, restando sólo el $42 \%$ para el resto de partidas de gastos del GAD Municipal de Salinas, conforme se observa en la figura 1.

Durante el periodo 2010 a mayo del 2014 la vía de ingreso al cantón, y el malecón de Salinas estaban llenos de vallas publicitarias las cuales eran generadoras de ingresos, pero estas no fueron parte del presupuesto institucional; hasta noviembre del 2013, existían aproximadamente 80 obreros en avanzada edad, con un sueldo promedio de 600 dólares c/u, quienes por insuficiencia de recursos económicos hasta diciembre de 2013, no eran parte del presupuesto institucional para efectos de liquidación; en similar situación existen 50 empleados con nombramiento, sueldo promedio 650 dólares.

El presupuesto de ingresos, por lo general, es liquidado anualmente con cartera vencida, debido a qué hasta abril de 2014, no se formulaban ni aplicaban políticas idóneas de gestión de cobro. El servicio de alcantarillado público atiende al $37 \%$ del total de las viviendas ubicadas en el área urbana, y 30\% del área rural, en estos sectores utilizan letrinas, pozos sépticos o pozos ciegos, no construidos técnicamente, 
ocasionando la contaminación del suelo; en la parroquia José Luis Tamayo, existe la laguna de oxidación, muy cercana a sectores residenciales, y sin ninguna clase de mantenimiento, produciendo contaminación por la emanación de olores debido a la falta de arborización y en su implantación no se consideró la dirección de los vientos.

Hasta mayo de 2014, el servicio de recolección de desechos sólidos (basura) se lo realizaba en forma irregular y con vehículos deteriorados por el escaso mantenimiento; existían solares vacíos, llenos de maleza, donde las personas hacen sus necesidades biológicas, convertidos en basureros y guarida de malhechores. Los centros de salud carecen de profesionales para atención las 24 horas del día y sin los equipos necesarios para el desarrollo de las actividades; la ciudadanía constantemente presenta su insatisfacción por el servicio recibido.

\section{RESULTADOS}

Tabla 4 Relación necesidades PDOT e inversión en obras públicas, cantón Salinas 2010 - 2016

\begin{tabular}{|c|l|c|c|c|}
\hline \multirow{2}{*}{ Ord. } & \multirow{2}{*}{ Denominación } & \multicolumn{2}{|c|}{ Ejecutado } & \multirow{2}{*}{ Total } \\
\cline { 3 - 4 } 1 & Demanda de necesidades & $\mathbf{2 0 1 0 - 2 0 1 5}$ & $\mathbf{2 0 1 6}$ & \\
\hline 2 & Inversión en obras públicas & 15.260 .644 & 3.409 .157 & $\mathbf{1 8 . 6 6 9 . 8 0 1}$ \\
\hline $\mathbf{3}$ & Déficit / Ssuperávit:... & $\mathbf{1 3 . 3 7 0 . 6 1 8}$ & $\mathbf{2 . 3 8 8 . 1 7 6}$ & $\mathbf{1 5 . 7 5 8 . 7 9 4}$ \\
\hline $\mathbf{4}$ & Coefi.rela: IOP/DDN & $\mathbf{5 3 \%}$ & $\mathbf{5 9 \%}$ & $\mathbf{5 4 \%}$ \\
\hline $\mathbf{5}$ & Coefi.rela: DOP/DDN & $\mathbf{4 7 \%}$ & $\mathbf{4 1 \%}$ & $\mathbf{4 6 \%}$ \\
\hline
\end{tabular}

Fuente: cédulas presupuestarias del GAD Municipal Salinas

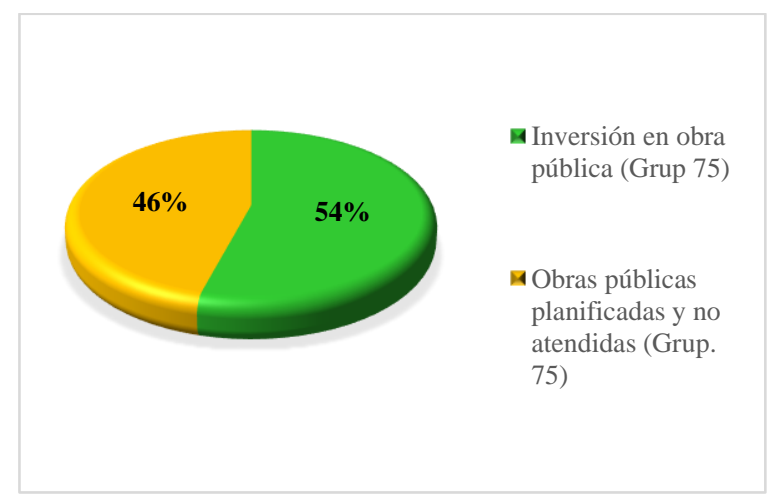

Figura 2 Necesidades según PDOT, atendidas y no atendidas

Los resultados de la investigación respecto a la relación necesidades según el Plan de Ordenamiento Territorial "PDOT" e inversión real en obras públicas, ilustrados en la tabla 4 y gráfica 2 , indican que el período 2010 - 2015 registró una demanda igual a \$28'631,262 y una atención a estas necesidades por \$15’260,644, determinando un coeficiente de relación inversión en obra pública versus demanda de necesidades, equivalente al $53 \%$.

En el 2016 la demanda de obras públicas "DOP” ascendió a \$5’797,333 y se ejecutó una inversión real en obra pública “OP” por \$3'409,157, representando el $59 \%$ del total de la planificación para este año. Los valores totales 2010-2016 indican que el 54\% de estos fueron invertidos en OP, interpretándose que de la suma total entre las demandas registradas en el PDOT 2011-2016 (solo hasta el 2013) y PDOT 2014-2016, el $46 \%$ no fue atendido.

Para el año 2011, se determina que se realizaron desembolsos por inversión en obra pública mayores a los valores previamente programados, situación que evidencia la inobservancia de la etapa "reformas presupuestarias".

Tabla 5 Relación de la inversión en obras públicas e ingresos efectivos, en dólares, período 2010 - 2016

\begin{tabular}{|c|l|r|c|c|c|}
\hline \multirow{2}{*}{ Ord. } & \multirow{2}{*}{ Denominación } & \multicolumn{3}{|c|}{ Años } & \multirow{2}{*}{$\%$} \\
\cline { 3 - 5 } 1 & $\mathbf{2 0 1 0}-\mathbf{2 0 1 5}$ & $\mathbf{2 0 1 6}$ & Total & \\
\hline \multirow{2}{*}{2} & Ingresos efectivos & 101.255 .982 & 18.425 .479 & 119.681 .461 & 100 \\
\cline { 2 - 5 } & Inversión en obras públicas & 15.260 .644 & 3.409 .157 & 18.669 .801 & 16 \\
\hline $\mathbf{3}$ & IOP con respecto al IE & $\mathbf{1 5 \%}$ & $\mathbf{1 9 \%}$ & $\mathbf{1 6 \%}$ & $\mathbf{1 6 \%}$ \\
\hline \multicolumn{3}{|l|}{$\begin{array}{l}\text { IOP = Inversión en Obra Pública } \\
\text { IE = Ingreso Efectivo }\end{array}$} \\
\hline
\end{tabular}

Fuente: cédulas presupuestarias del GAD Municipal de Salinas

Adicionalmente, en la tabla 5 se observa que el coeficiente de relación "Inversión en Obra Pública versus Ingreso Efectivo", periodo 2010 - 2015, representa el 15\%; 2016, $19 \%$ y en totales $16 \%$.

Tabla 6 Relación de necesidades según PDOT e inversión en obras públicas, cantón Salinas, en dólares, período 2014. 2016

\begin{tabular}{|c|l|c|}
\hline \multirow{2}{*}{ ORD. } & \multicolumn{1}{|c|}{ DENOMINAC IÓ N } & PERÍODO \\
\cline { 3 - 3 } & & $\mathbf{2 0 1 4 - 2 0 1 6}$ \\
\hline 1 & Demanda de necesidades (DN) & 17.892 .000 \\
\hline 2 & Inversión en obras públicas (IOP) & 7.426 .590 \\
\hline 3 & Deficit de obras públicas (DOP) & 10.465 .410 \\
\hline 5 & Coeficiente de relación: IOP/DN & $42 \%$ \\
\hline & Coeficiente de relación: DOP/DN & $58 \%$ \\
\hline
\end{tabular}

Fuente: documentos y cédulas presupuestarias del GAD Municipal Salinas

Durante el periodo 2014 a 2016, la partida 75 inversión en obra pública, presenta registros de pagos realizados por $\$ 7^{\prime} 426,490$ equivalentes al $42 \%$ de 
$\$ 17^{\prime} 892,000$ y un déficit de $\$ 10^{\prime} 465,410$ $\left(\$ 17^{\prime} 892,410-\$ 7^{\prime} 426,490\right)$ correspondiente al $58 \%$ del valor de las demandas de necesidades consideradas en el PDOT 2014-2019, no atendidas por insuficiencia de recursos monetarios.

Dentro de la investigación también se analizó la partida presupuestaria número 38 denominada "Cuentas Pendientes de Cobro"

Tabla 7 Comportamiento anual de la partida presupuestaria "Cuentas pendientes por cobrar" en dólares, años 2010 a 2016

\begin{tabular}{|c|c|c|c|}
\hline AÑOS & SALDOS AL 31-DIC & VARIACIÓN & $\%$ \\
\hline 2010 & 935.568 & & \\
\hline 2011 & 1.486 .426 & 550.858 & $59 \%$ \\
\hline 2012 & 2.931 .562 & 1.445 .137 & $97 \%$ \\
\hline 2013 & 2.933 .204 & 1.641 & $0,06 \%$ \\
\hline 2015 & 1.239 .429 & -1.693 .775 & $-57,74 \%$ \\
\hline 2016 & 1.218 .937 & -20.492 & $-1,65 \%$ \\
\hline
\end{tabular}

Fuente: documentos y cédulas presupuestarias del GAD Municipal Salinas

Las cuentas pendientes de cobro al 31 de diciembre del 2010 registraron un saldo acumulado de $\$ 935,568$; cerrando el 2011 con un incremento del 59\%; 2012 con el 97\%; en el año 2013 los derechos al cobro no tuvieron cambios relevantes; 2014 experimentó una recuperación de cartera del 57,74\%; 2015 el comportamiento fue con tendencia mínima a la baja; para al 31 de diciembre del 2016 volverse a elevar en 142,95 puntos porcentuales con respecto al 2015, conforme se ilustra en la table número 7.

Para este lapso de tiempo, equivale decir que la inversión destinada para contribuir al alcance de mejores niveles de vida en la población del cantón Salinas, conforme lo establece el Plan Nacional del Buen Vivir, está por debajo de lo requerido en este destino turístico del Ecuador y del Pacífico Sur.

\section{Conclusiones}

El presente estudio y análisis evidencia que la hipótesis planteada "Los Ingresos del Gobierno Autónomo Descentralizado Municipal de Salinas (GADMS), no permiten satisfacer las necesidades básicas de la población del Cantón”, es Verdadera, en razón de que los ingresos destinados a la inversión pública son menores a lo que realmente demanda la sociedad del cantón Salinas.
El catastro municipal estuvo sin actualizarse desde el 2007 hasta enero del 2015 que se realizó el correspondiente estudio, el cual fue aprobado por el Consejo Municipal, para entrar en vigencia a partir de enero de 2016. y como resultado del cumplimiento del artículo No. 139 del Código Orgánico de Ordenamiento Territorial, Autonomía y Descentralización, mediante el cual se establece que es obligación de los gobiernos autónomos descentralizados municipales actualizar cada dos años los catastros y valorar la propiedad urbana y rural, se tiene: al 31 de diciembre del 2016 según estados financieros, la sumatoria de los rubros impuestos, tasas y contribuciones, registraron una variación en más del 17\% y $13 \%$ con respecto al 2014 y 2015 en el respectivo orden.

La tasa anual de funcionamiento de establecimientos turísticos (hoteles, hostales, pensiones, bares, discotecas, fuentes de soda, peñas, centros de convenciones, restaurantes, cafeterías, agencias de viajes y turismo), regulada con ordenanza publicada en el Registro Oficial No. 38 del 12 de marzo del 2003, no ha sido actualizada hasta la presente fecha.

La ejecución presupuestaria de gasto en personal es alta, en el año 2012 ascendió a \$7’764,902.47, sumatoria de $\left(\$ 2^{\prime} 659,474.93+\quad \$ 5^{\prime} 105,427.54\right)$, representan el 50\% de $\$ 15^{\prime} 672,896$ (Quince millones seiscientos setenta y dos mil ochocientos noventa y tres 00/100 dólares), $100 \%$ del presupuesto.

La inversión pública en el año 2012 ascendió a $\$ 2^{\prime} 407,893$ y constituye el $15,36 \%$ de $\$ 15^{\prime} 672,893$ (Quince millones seiscientos setenta y dos mil ochocientos noventa y tres 00/100 dólares), $100 \%$ del presupuesto para este año, observándose una distribución inequitativa del ingreso.

Del $100 \%$ de ingresos efectivos generados durante el periodo 2010-2016, apenas se destinó el 15,60\% para inversión en obra pública, parte proporcional del presupuesto institucional que solo cubre el $54 \%$ del total de necesidades debidamente planificadas en los PDOT correspondientes, quedando el $46 \%$ sin atender.

La mayor recaudación de los ingresos de toda entidad pública de esta naturaleza, está en función de las iniciativas y grado de responsabilidad de los gerentes públicos y directivos, de las políticas internas de gestión de cobro que se implementen y de la dirección efectiva e integrada de los procesos de creación de valor público (inversión), a fin de optimizarlo asegurando la máxima eficacia, eficiencia y efectividad de su desempeño (presupuesto), la consecución de los objetivos institucionales y la mejora continua de los resultados de las administraciones de turno. 
La gestión en la administración de ingresos y gastos del sector público, es una estrategia aplicada dentro de estructuras orgánicas con disciplina fiscal, es decir, que los gerentes, directivos y todo servidor público, deben ser profesionales capaces y fiel observadores de leyes y normativas. El presupuesto de ingresos por lo general es liquidado anualmente con cartera vencida, por lo que no se formulan ni aplican políticas idóneas de gestión de cobro.

\section{Recomendaciones:}

Formular, implementar y ejecutar políticas idóneas de gestión de cobro.

Gestionar una distribución equitativa del ingreso efectivo, direccionando mayores recursos a la inversión en obras públicas.

Preparar el plan anual y plurianual de inversiones, conforme lo estipula el Código Orgánico de Finanzas Públicas. 202

Administrar los recursos del Estado dentro de un contexto de disciplina fiscal.

\section{Bibliografía:}

Asamblea Nacional. (20 de octubre de 2008).

Constitución de la República del Ecuador. Quito, Pichincha, Ecuador.

Basavilbaso, E. (2011). Gestión por Resultados en la Administración Pública. Buenos Aires.

Corporación de Estudios y Publicaciones. (Febrero de 2012). Código Orgánico de

Organización, Autonomía y

Descentralización. Quito, Pichincha,

Ecuador: Taller de la Corporación de

Estudios y Publicaciones.
Corporación de Estudios y Publicaciones. (Abril de 2012). Código Orgánico de Planificación y Finanzas Públicas, Tomo III. Manual General de Contabilidad Gubernamental. Quito, Pichincha, Ecuador: Taller de la Corporación de Estudios y Publicaciones.

Corporación de Estudios y Publicaciones. (Febrero de 2013). Código Orgánico de Planificación y Finanzas Públicas, Tomo I. Estudio y Análisis de COPLAFIP. Quito, Pichincha, Ecuador: Taller de la Corporación de Estudios y Publicaciones.

Ecuador, M. d. (2011). Directrices de la Programación Presupuestaria 2011-2014. Quito, Pichincha, Ecuador: Secretaría Nacional de Planificación y Desarrollo.

García Moreno , M., \& García López, R. (2011). Gestión para Resultados en el Desarrollo en Gobierno Subnacionales. INDES y BID.

Guzman, M. (2011). Gestión para resultados en el desarrollo en gobiernos subnacionales. Presupuesto por resultados. Quito, Pichincha, Ecuador: INDES o PRODEV.

Herrera Library, F. (2007). Modelo Abierto de Gestión para Resultados en el Sector Público. Wahington: Banco Interamericano de Desarrollo.

Panchana P.Margarita (24 de nov de 2014). Los Ingresos del GADMS y el gasto en la Inversión Pública, periodo 2007-2012, Guayaquil, Guayas, Ecuador.

Paredes, F. (2006). Presupuesto público: Aspectos teóricos y prácticos. Mérida Venezuela: Editorial Venezolana C.A.

Secretaría Nacional de Planificación y Desarrollo SENPLADES. (24 de junio de 2013). Plan Nacional del Buen Vivir, 2013-2017. Quito, Pichincha, Ecuador: SENPLADES. 